\title{
A163: Alternative Mechanisms of Care Delivery in Pediatric Rheumatology: To What Extent Do They Expand the Reach of Pediatric Rheumatologists?
}

\author{
Meredith P. Riebschleger, Joshua Tootoo, Amanda Mroczek, and Sarah Clark
}

Background/Purpose: Pediatric rheumatology (PR) practitioners are concentrated in academic centers, rather than being evenly distributed throughout the US. Alternative mechanisms of care delivery, such as secondary clinic sites and telemedicine, may function as a bridge between PR practitioners and children who live far from academic centers. The objective of this study was to determine the prevalence and distribution of alternative mechanisms of care delivery in US PR practices.

Methods: PR practices were identified using the American College of Rheumatology member directory and the Children's Hospital Association hospital directory. Practice websites were reviewed to obtain data regarding secondary clinic sites and use of telemedicine. Practices were then called to confirm website data and obtain data not available on websites. Distance between each main and secondary clinic site was calculated. Geographic information system (GIS) was used to integrate study data with census data.

Results: $77 / 90$ PR practices participated in phone calls and were included in analyses. 46 practices $(60 \%)$ reported using secondary clinic sites; the number of secondary sites per practice ranged from 1-6 (total 96

University of Michigan, Ann Arbor, MI. sites). Frequency of clinics at secondary sites ranged from weekly to once per year; $>80 \%$ of sites held clinics at least once each month. Distance from the main practice site to secondary clinic sites ranged from 6.2 to 2265 miles, with $65 \%(62 / 96)<60$ miles from the main practice site and almost half $(47 / 96)<30$ miles from the main practice site. Census data indicate that $39 \%$ of US children live $>60$ miles from a main practice site and $30 \%$ of US children live $>60$ miles from any PR clinic site. Of the 12 states without a PR practice, 3 were served by secondary clinic sites. Seven practices $(9 \%)$ reported telemedicine capability, but only $3(4 \%)$ reported ever using it.

Conclusion: Though most PR practices utilize secondary clinic sites, nearly $2 / 3$ of those sites are $<60$ miles from the main practice site and nearly $1 / 3$ of US children live far ( $>60$ miles) from any PR clinic site. Few practices currently use telemedicine. Therefore, it is not clear that secondary clinic sites and telemedicine, as currently employed, significantly expand the reach of PR practitioners. Future studies should investigate the efficiency and effectiveness of alternative mechanisms of care delivery, to determine how best to serve children who live far from academic centers.

Disclosure: M. P. Riebschleger, None; J. Tootoo, None; A. Mroczek, None; S. Clark, None. 